\title{
AN ILLUMINATED PERIMETER WITH CAMPIMETER FEATURES.
}

\section{(. V. Ferrel: and (iertrude Raxi).}

BRYX MAWR COLLEGE.

After discussion of the conditions to be met in designing an accurate and serviceable instrument both as to illumination, background, steadiness of eye and fixation, the instrument worked out is here described and illustrated.

This apparatus was devised in response to a request from a committee appointed by the American (Ophthalmological Society to work out a better standardization of the illumination of perimeters and test charts. The request was for a feasible means of illuminating the perimeter arm with light of a good intensity and quality, so that every print on the arm in any meridian in which it may be placed shall receive equal intensities of light. Intensity and quality of illumination, however, are only two of the factors which influence the results of the perimetric determination. In devising the instrument described in this paper, it has been our purpose to provide a control also of other factors which are of importance to the work of the office and clinic. The perimeter devised and to be described in this paper has been adopted by the Committee as the standard instrument for office and clinic work.

The variable factors which influence the apparent limits of color sensitivity are. so far as we have been able to discover, the wave length and purity of the stimulus, the intensity of the stimulus and the visual angle, length of exposure of the eye, the method of exposture (moring or stationary stimulus), accuracy and steadiness of fixation, the intensity of the general illumination of the retina and its state of adaptation, breadth of pupil, and the brightness of the preexposure and of the background or surroundingr field. The most important of these from the standpoint of the office or clinic are perhaps the intensity of the stimulus, the brightness of the preexposure and the surrounding field, the intensity of the general illumination, and the accuracy and steadiness of fixation.

'erhaps errors in refraction should be included in the above list of factors. They differ from those factors, how- ever, in that they are a source only, or mainly at least, of differences in breadth of field between observers, not for the same observer at different times. They are not therefore a serious source of trouble in the use of perimetry to check up the advance or recession of a given pathologic condition, but they are confusing in diagnosis. We have no certain means of telling, for example, how much of the results in any given case of high myopia is due to the refractive condition and how much if any is pathologic. To add to our knowledge on this point we are now conducting an investigation to determine the effect of various amounts and kinds of refractive errors on the results of ficld taking. We can not help but feel, as is stated and discussed in the paper, that a provision should be made in our practice of field taking to include one set of results under the correcting glasses, even tho it means either working under conditions which give a narrow field or being content with results which do not include the wider portions of the temporal field.

(1) Intensity of stimulus. By a sufficiently wide variation of this factor alone, the zones of color sensitivity may be made to have almost any breadth within the limits of the field of vision, to differ radically in shape. and even to change or reverse their order of ranking as to breadth. When pigment surfaces are used as stimuli, the illumination of the perimeter arm determines the intensity of the stimulus light. Two methods are proposed for securing an even illumination of the stimulus at every point on the perimeter arm and of reproducing this illumination from time to time.

Method I. When the source of light is inlaid in the surface of the arm or its continuation, the illumination on this surface will be equal for approxi- 
mately $180^{\circ}$ on either side of the source. The value of this illumination at every point will be equal to the normal flux of light from the luminous surface divided by four times the square of the radius of curvature of the perimeter arm, or four times the square of the distance of the eye from the perimeter arm. A perimeter embodying this principle of illumination is being constructed in the following way. A lamp house is fastened on the continuation of a $90^{\circ}$ arm in such a posi-

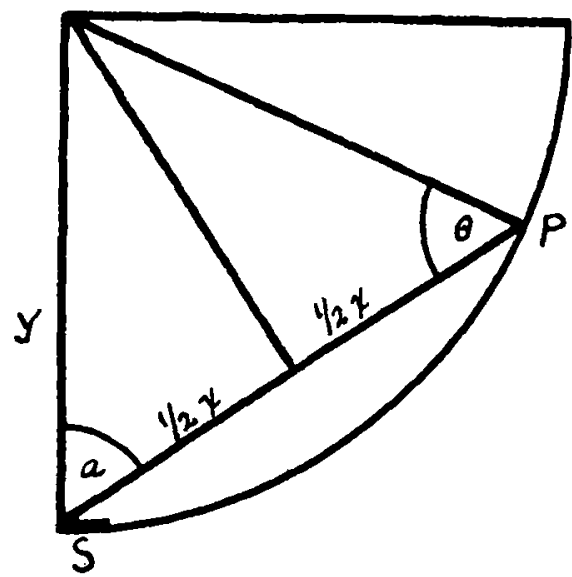

Fig. 1. -1), monstration of even illumination of perimeter arm.

tion that an opening in its surface facing the observer lies in the continuation of the surface of the perimeter arm. This opening is filled in with diffusing glass bent to take the curvature of the arm, and shaded in such a way as to shield the eye of the physician and the observer without changing or interfering with the distribution of light to the perimeter arm. The lamp house rotates with the arm and thus illuminates it uniformly at every point in all meridians.

The principle by which an even illumination of the perimeter arm is secured by this method may be demonstrated as follows: I et S, Fig. 1, be the source of light inlaid in the surface of the arm; $P$ any point on the perimeter arm that is to be illuminated; $X$ the distance from $S$ to $P$; $v$ the radius of curvature of the arm or the distance of the eye from the arm; $a$ the angle of emission of the light from the source of $S$; and $\theta$ the angle of incidence of this light at the point $P$. Then the intensity at $P$ will be inversely as the square of the distance of $\mathrm{P}$ from $\mathrm{S}$ or inversely as the square of $X$, and directiy as the cosine of the $1 / 2 x^{2}$

angle of emission $a,--$, and of the angle of incidence $\theta$, also $\frac{1 / 2 x^{2}}{y}$. That is,

$I_{1}=I \times \frac{1}{x^{2}} \times \frac{I / 2 x}{y} \times \frac{I / 2 x}{y}=I \frac{I / 4 x^{2}}{x^{2} y^{2}}=\frac{I}{4 y^{2}}$ in which $I_{1}$ is the intensity of light at $P$ and $I$ the intensity at $S$. From this equation is derived the law of illumination of the arm: the intensity of light at any point on the arm is equal to the normal flux of light from the source divided by four times the square of the radius of curvature of the arm.

The method has the following objections. (1) The difficulties in construction are not easy to overcome.

Evenness of illumination requires an approximately perfectly diffusing glass. This glass is difficult to obtain and prepare and its transmission is apt to be low. Moreover the light incident on the perimeter arm should approximate daylight in composition. The selective $a b$ sorption required to correct the light from the lamp to this composition further reduces the intensity enormously. This double loss, first by absorption, and second by the somewhat wasteful type of distribution employed, renders it difficult to get an adequate intensity of illumination of the perimeter arm.

Mcthod 2. When the source of light lies on the perpendicular to the plane of the perimeter arm at its center of curvature, it will be equidistant from every point on the arm; also the angles of emission and incidence of the beam of light will be equal for every point on the arm. A perimeter has been constructed embodying this principle of illumination. This perimeter is shown in Figs. 2-5. Two arcs of the same radius of curvature were constructed at right angles to each other; one a $180^{\circ}$ arc, the perimeter arm; the other a $90^{\circ}$ arc, the lamp arm, at the end of which is placed the source of 
light. In order that the source of light shall sustain a fixed relation to the perimeter arm for all positions of that arm, the two arms are fastened together at the center of rotation. About the source is a housing which was designed in such a way as to shield the eye of the patient and the physician without interfering with the distribution of light to the

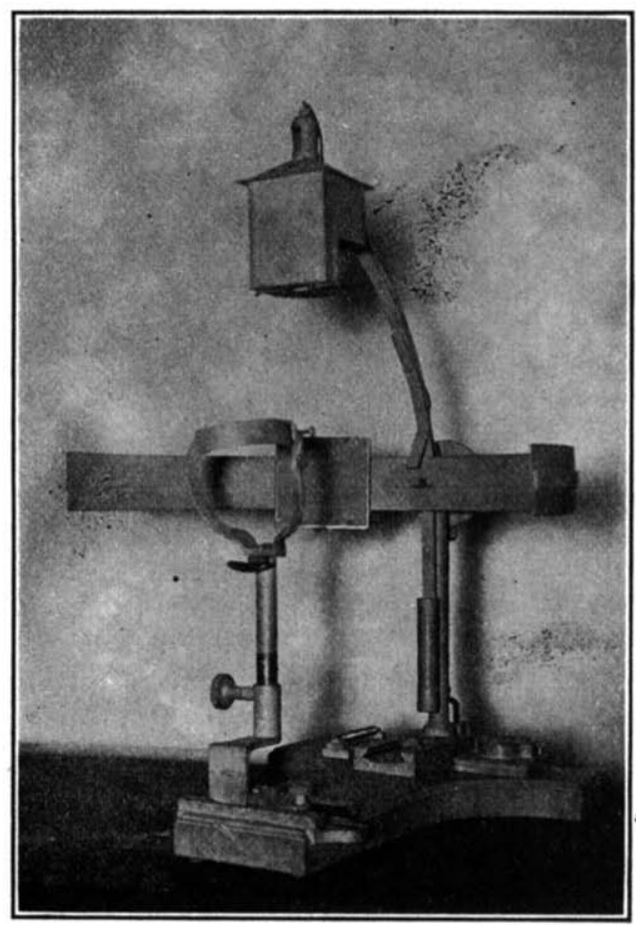

Fig. 2,-Perimeter with illuminating lamp above and target upon the arc.

perimeter arm. This housing is made of black japanned iron and is painted a mat black on the inside in order that as nearly as possible all of the light which passes to the perimeter arm shall radiate directly from the lamp filament. Its dimensions are $4 \frac{1}{4} \times 4 \frac{1}{4} \times 5$ in. A rectangular aperture $2 \mathrm{r} / 8$ in, wide was cut out of the side of the housing facing the perimeter arm, at the bottom and for 3 in. back on the two adjacent sides. The relation of the lamp to the aperture of this housing is such that the light radiates freely without shadows from the filament to every point on the perimeter arm. In order that the lamp.may be re- moved when desired, the bottom of the housing is hinged at the back and is held in place by a latch on either side. To prevent overheating, the housing is well ventilated by especially designed lighttight ventilators, four in the sloping roof of the lamp house and four on each of the sides at the bottom.

Provisions are made in the construc-

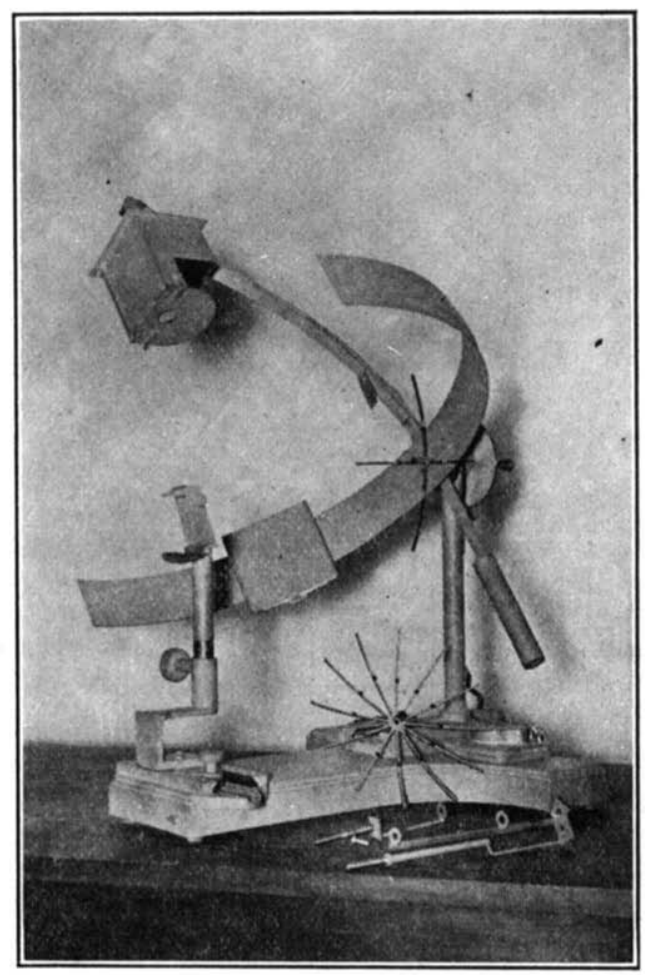

Fig. 3.-Perimeter with arc and lamp swung obJiquely. Arms for mapping central scotoma and
sights detached.

tion of the lamp house for filtering the light to daylight quality. This is accomplished in two ways. (a) A well seasoned 75 watt type $C_{2}$ (blue bulb) Mazda lamp operated by ammeter and rheostat control is used as the source of light. This is the ordinary blue bulb commercial lamp and gives only approximate daylight quality. The surface of the bulb is acid-etched to diffuse the light. The intensity of light incident on the perimeter arm when this lamp is used as source of light is 17 foot-candles. (b) A type C (clear bulb) Mazda lamp of 75,100 or 1.50 watts depending upon the intensity of light desired, is used a: 
the source. The light from the lamp is filtered to daylight quality by means of a double-etched collar or cylinder of carefully prepared daylight glass $2 \frac{1}{2}$ in. broad and 4 in. in diameter, which can be inserted into the bottom of the lamphouse so as to fill completely the threesided aperture. When in position all of the light emitted from this lamp house

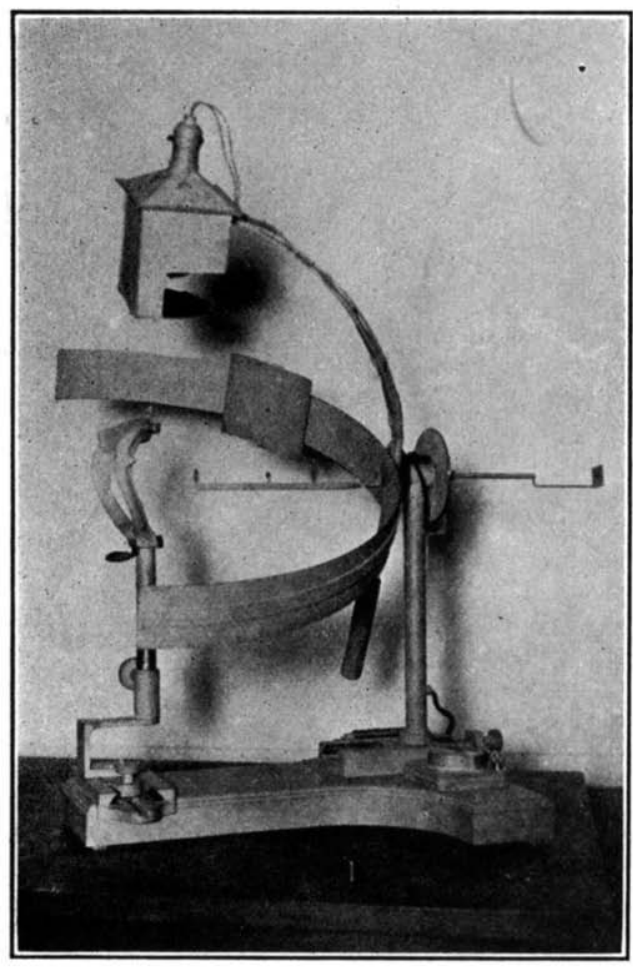

Fig. 4.-Side view of perimeter with attachments in place.

must pass thru the filter. The collar is securely held in position by three pins so placed in the bottom of the lamp nouse as to form the apices of an equilateral triangle of the appropriate dimensions. This bottom is hinged so that it can be dropped down for the insertion of the collar and is provided with latches, so that it can be held securely in position when raised. The light of a type $C$ Mazda lamp filtered by this glass is a very close spectrophotometric approximation to north skylight. The coefficient of transmission of the glass is 15 percent. The light incident thru the filter on the perimeter arm from a 100 watt type C Mazda lamp has a value of 2.6 foot-candles, an intensity very suitable for the taking of a low illumination field, the importance of which in diagnosis will be discussed later. Higher intensity fields may be obtained by using higher wattage lamps.

This perimeter is not difficult to construct or to operate. It provides for a

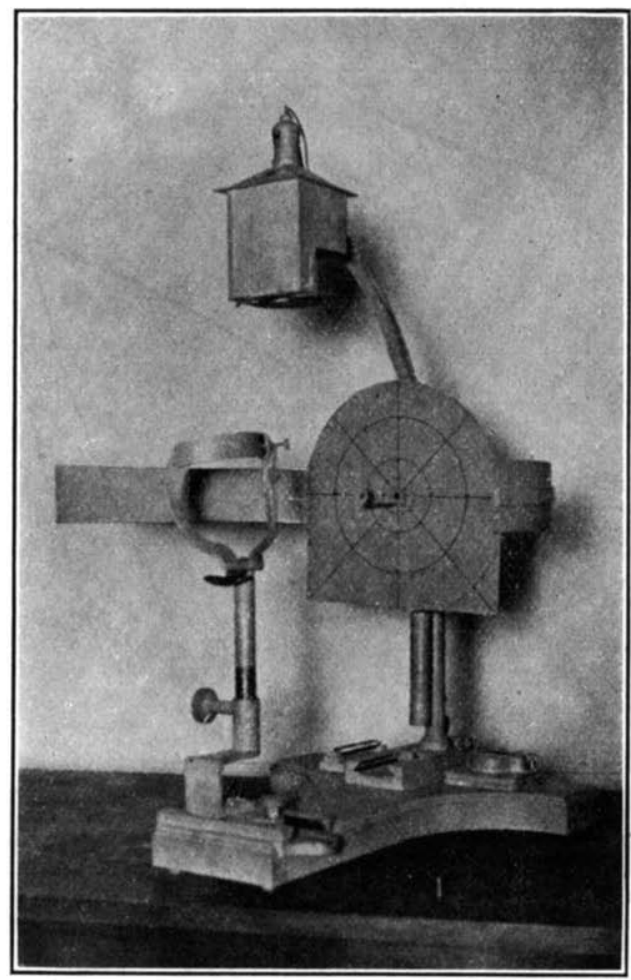

Fig. 5.-Oblique view of perimeter, showing campineter field and sight in piace.

uniform illumination of the perimeter arm in all meridians with light of a good intensity and quality; and with it a precision of control is possible which is comparable with the work of the physical laboratory. Of the two instruments we have devised, it is without doubt much the more feasible, and it is also very probably the more correct in actual practice. Both instruments are correct in theory.

(2) The brightness of the preenposure and the surrounding field. The brightness of the surface to which the eye is preexposed may change the appafent limits in certain meridians as 
much as $17-20^{\circ}$. A preexposure lighter than the color gives a dark, and one darker than the color a light after image. These after images change profoundly the saturation of the color sensation, also its hue. A background or surrounding field lighter or darker than the color produces a similar effect on the limits, but not so great. In this case the disturbing achromatic effect is due to physiologic induction or contrast. The variable effect of brightness of preexposure and surrounding field can be eliminated only by making both a gray of the same brightness as the stimulus color. Here again a precise control of the intensity of the illumination for all points on the perimeter. arm becomes important. That is, the shade of gray which is needed to match the color in brightness changes with change of illumination; therefore, the selection of a gray which will match the color in brightness for all points of work presupposes constancy and uniformity of illumination. A further advantage is gained by making the background of the same brightness, as the color. That is, when color and background are of the same brightness the stimulus disappears completely when the limit of sensitivity to that color is reached, instead of turning into a gray, concerning the colorlessness of which the patient is apt to be in doubt. This gives the effect of the disappearance type of photometer and like it adds greatly to the ease and certainty of making the judgment.

The control of brightness of preexposure and surrounding field is provided for in the following way. To the stimtulus carriage is attached a light aluminum holder, $\mathrm{N}_{\text {o. }} 19$ B \& S gauge, grooved to hold a card $5 \times 6$ in. These cards are covered on one side respectively by grays of the brightness of the four colors, red, yellow, green and blue of the Hering standard series of pigment papers, as seen in the peripheral retina. At the center of each of these cards is pasted a disc of the appropriate color stubtending a visual angle of $1^{\circ}$. 'To provide for the control of the preexposure for the stationary method of giving the stimulation, cards identical with the background cards are provided, covered also on one side with a gray of the brightness of the color. The stimulation by this method is given as follows: The stimulus is placed at the point to be tested and covered with the preexposure card. The observer is told to take his fixation. At a given signal the stimulus is uncovered for one second and recovered. In case the moving stimulus method is used, the surrounding field serves as the preexposure.

The perimeter arm and body are painted a gray of a shade which is approximately mid-gray to the blue and yellow, the darkest and lightest of the stimuli employed. In our own laboratory the perimeter is used on a table painted with the same gray and stands before a gray screen. These latter precautions, however, are not necessary.

When the instrument is supplied to the profession, provision will be made that the stimulus and preexposure cards, a seasoned lamp, and all other perishable parts can be furnished in the quantities desired.

(3) 'The accuracy' and steadiness of firation. All are familiar with the disturbing effect of inaccuracy and unsteadiness of fixation. If correct and reproducible results are to be obtained, the eye must be accurately placed at the center of the sphere in the surface of which the perimeter arm lies, and the line of sight must not shift from the fixation point while the color observation is being made. As an aid to the correct placement of the eye and a check on its steadiness of fixation, two devices have been provided. (a) A small circular mirror is used as a fixation object in which the observer sees the image of his own cye (Fig. 2). When the eye is correctly placed with the line of sight normal to the surface of the mirror at its central point, the fact is indicated to the observer by the position of the image of his pupil and iris as scen in the mirror. Not only is this simple device of service in determining the correct position of the eye, but it aids the observer greatly in holding a steady fixation.

We scarcely need to point out that a fixation object does not afford an accurate control of fixation. Exact checking methods show that the patient is not 
always fixating the object when he thinks he is. The only guide to monocular fixation is clearness of seeing, and this is a criterion that presents considerable latitude. However with a mirror, the patient has an objective check on the position of his eye.

One of the objections to the use of a mirror as a means of controlling fixation is the likelihood of glare from its surface, probably due to a combined specular and diffuse reflection rendering, in proportion as it occurs, the clear seeing of the image of the eye by the patient unnecessarily difficult. This objection has been obviated in the instrument described by cutting off the direct radiations from the lamp from the mirror by a narrow shield, which can be turned back out of the way when not in use. With the shield in position the eye receives from the mirror only the light which is first reflected from the eye to the mirror and then back to the eye. The device adds greatly to the ease and clearness with which the patient sees his imaged eye. The climination of the troublesome glare on the surface of the mirror is rendered particularly simple and easy in case of this instrument because of the plan of illumination employed, $i$. $e$, the light all comes from a fixed source above and directly in front of the mirror. If the perimeter were illuminated from a window, for example, and its position with reference to the window changed as is needed in order to give as nearly as possible equal illumination of the stimulus in its various positions, the elimination of glare from the surface of the mirror would not be so simple a problem.

Another theoretic objection to the mirror is that the clear seeing of the iris in the mirror requires the eye to focus for twice its distance from the perimeter arm. When this distance is $33 \mathrm{~cm}$., as in the present case, the use of the mirror as a fixation device throws the refracting system of the eye 1.50 'diopters out of focus for the colored stimulus on the perimeter arm. Just how important this is when the problem is the mapping of the limits of color sensitivity and not a determination of acuity is difficult to say a priori. The poor imaging for the peripheral field is well known to all who have made determinations of acuity at points far removed from the center of the field. The additional blurring due to an error of focussing of the magnitude described is probably of negligible consequence at points having an excentricity of $30-70$ degrees, particularly in determinations of color sensitivity. In any event, we have not been able to detect appreciable differences in the limits of sensitivity at 17 foot-candles of illumination with the mirror as a fixation device and with the other device provided, in a case where the eye is correctly focussed for clear seeing at $33 \mathrm{~cm}$.

(b) The second device for the control of fixation is similar in principle to a peep-sight and may be called a parallax or peep-sight device (Fig. 5). $\Lambda$ small disc or button $(8 \mathrm{~mm}$. in diameter $)$ at the center of rotation of the perimeter arm is viewed thru a circular opening $(7 \mathrm{~mm}$. in diameter) in a thin round metal disc $9 \mathrm{~cm}$. nearer to the eye. The plane of the disc and the viewing opening are both normal to the line of sight when the eye has its correct position and fixation. When the eye has this position and fixation, the relation of size of disc and opening is such that the disc is seen not quite to fill the opening. The disc is painted black, also the edge of the opening; thus when the eye has the proper position and fixation, the edge of the opening is seen concentric to the disc, with a narrow ring of the gray of the brightness of the perimeter arm between. The control afforded by this device is very sensitive. A very slight deviation of the position or fixation of the eye results in the complete or partial extinction of this ring at a point in the direction of the deviation. Iike the mirror the device is mounted on a short pin or plug which can be inserted in the fixed tubular axle about which the perimeter arm rotates.

However, while the image in the mirror or the correct sighting of the fixation disc thru its opening will indicate to the observer when the line of sight is normal to the surface of the fixation object at its central point, there are two important features in the correct adjustment of the eye over which these devices exercise no control: (a) the distance of 
the eye from the mirror; and (b) the agreement of the meridians of the field of vision as read on the perimeter with the meridians of the retina. In order that it may be known when the eye is at the correct distance from the perimeter arm, a light measuring rod $33 \mathrm{~cm}$. in length is provided, to one end of which is fastened at right angles a small metal disc. In making the adjustment for distance one end of the rod is placed against the mirror at its center and the distance of the perimeter from the observer's eye is changed by means of the coarse screw adjustment to be described later, until the closed lid is $i$ ust in contact with the metal disc.

Perhaps the simplest device for ensuring a constancy of relation of the meridians of the retina to the meridians of the field of vision as laid off by the perimeter arm, in other words for guarding against a slight tilting of the head to one side or the other, is a mouth bit. We have designed (Fig. 3) a very small and unobjectionable mouth bit of light wood to be changed for each observer, so shaped that it can not be bitten too far forward or back, and thus the distance of the eye from the mirror be changed, or too far to one side or the other. There seems, however, to be an insuperable prejudice against the use of a mouth bit by both the physician and the patient. We have designed, therefore (Figs. 2, 4 and 5) a head rest which follows approximately the outlines of the forehead. side of the head and face, furnished with a suitably cupped chin rest, the height of which is adjustable, also its position forward or backward. To provide for individual differences in shape and breadth of forehead, an adjustable forehead piece or band of thin spring steel extending well around to the side of the head is screwed at its central point to the forehead piece of the head rest. This forehead band is adjusted to fit foreheads of different shape and breadth by.means of a set screw on either side near the two ends of the band. When the chin rest is adjusted to its proper height and the forehead band is made to fit the forehead, the patient's head is held comfortably in position and sufficiently rigid, it is believed, to satisfy the needs of office and clinic work. At least the probability of tilting the head to one side or the other, thus causing a disagreement of the meridians of the field of vision as indicated by the perimeter readings with the meridians of the retina is very greatly lessened, if not entirely obviated. This, it will be remembered, is the especial purpose of the device, the other features of the control being taken care of in other ways.

In order quickly and conveniently to locate the patient's eye at the center of the perimeter system, three adjustments are provided; a rack and pinion to raise and lower the head, a second rack and pinion to shift the head to right or left, and a coarse screw adjustment to change the distance of the perimeter arm from the eye. In the process of getting the eye in position, the patient bites the mouth bit or adjusts the head in the head rest, brings the eye to the level of the fixation device (mirror or peep-sight) with the first rack and pinion, and centers its image in the mirror with the second rack and pinion. The physician gets the correct distance of the perimeter arm from the patient's eye by means of the screw adjustment and the measuring rod already referred to. Once these adjustments are made for an eye, they need not be made again during the process of taking the fields for that eye; that is, the biting of the mouth bit or the return of the head to the head rest guarantees that the eye always returns to the same position for which the original adjustments were made.

$A$ very great practical need in a clinic perimeter is a method of controlling fixation for patients who have a central scotoma or pathologic blind area. With the eye properly adjusted for taking the fields, these patients are not able to see a central fixation object. A device has been constructed (Fig. 3) for controlling the fixation of such patients in the following way. The perimeter arm is made to rotate about a hollow fixed axle. Into this fixed axle telescopes the stem at the end of which is the mirror or peepsight used for patients with normal or approximately normal central vision, also a hollow stem carrying the device used to control the fixation of patients with a 
central deficiency. This latter levice consists of four light arms or rods at right angles to each other curved to follow the arc of the perimeter arm, and of sufficient length to provide for all probable breadths of scotoma. Each of these arms carries a small stimulus the distance of which from the center of the field is adjustable. In adjusting the patient's eye i)y this device, the physician looks thru a small telescope contained in the hollow tubular axle and lines up the pupil of the patient's eye with the cross hair in the field of the telescope. When the patient's eye is observed to be in position, two or all four of the stimuli as may be desired are adjusted so that they can just be seen by the patient at the exlges of the scotoma. These stimuli serve as the control of the patient's fixation, his instructions being to direct the eye so that all are visible.

This fixation device intended to control the fixation during the taking of the fields can be made serviceable for mapping the scotoma itself by adding 12 or more graduated arms equally spaced, making 16 in all, provided with stimuli similar to those already described. Then when the fixation is oltained by the adjustment of the four stimuli designed for that purpose, the further mapping of the scotoma is accomplished by moving the remaining 12 until they are on the edges of the scotoma. Or if desired the four stimuli designed for the control of the fixation may not be used for that purpose at all. The physician may watch the patient's eye thru the telescope directing the fixation by means of the cross hairs, while all of the stimuli are moved into position on the edges of the blind area. This objective control of the fixation may also be used, both in mapping the scotoma and in taking the ficlds when a central scotoma is present.

If desired the patient's eye can be rendered more visible by reflecting the light from the lamp directly on to it. This is provided for by placing a small oblong mirror of specular metal on the lamp arm at such a position and angle that the light received from the lamp will be reflected on to the iris. This mirror is hinged to the lamp arn and can be turned back against it when not in use. The hinge is provided with a stop so that when the mirror is turned down it can not go beyond the position required to reflect the light on to the eye. "The visibility of the eye in the field of the telescope is considerably increased also by excluding as far as possible scattered light. This was done by putting the objective lens well back in the tube, painting the inside of the tube a mat black and placing on the end of the tube nearer the eye a short tubular hood, which shields the opening from the direct rays from the lamp and admits only those reflected from the cye.

Another important need in a clinic perimeter is a method of giving the correct location and fixation to eyes suffering with high myopia. Eyes with myopia ranging from 8-20 diopters would have great difficulty in seeing a fixation object at a distance of $33 \mathrm{~cm}$. Because of the grave pathologic changes which take place in the retina and choroid of eyes suffering from high myopia, particularly in the region of the macula and nerve head, it is of great importance to be able to use both the perimeter and the tangent screen to be described later, in the examination of eyes in the more advanced stages of myopia. Three provisions have been made for this. (1) The mirror may be mounted on a rod sufficiently long to permit of its location at any point in the line of sight between the perimeter arm and the eye. This rod may be inserted in the tubular axle on which the perimeter arm rotates. (2) A peep-sight device is provided similar in principle to the one already described, and so constructed that it may suffice as a fixation control for values of myopia ranging between 8 and 20 diopters. As shown in Fig. 4, this device has been so constructed that it can be used either for the normal or the myopic eye. That is, it is provided with two sets of fixation targets and viewing openings, each at their proper distance from the eye. Both sets are hinged to the narrow steel bar which serves as their support. so that either can be turned down out of the way when not in use. The diameter of the target for use with the normal eye is $8 \mathrm{~mm}$.; the diameter of the viewing opening, $7 \mathrm{~mm}$.; and the distance of the 
opening from the target, $9 \mathrm{~cm}$. The diameter of the target for use with the myopic cye is $8 \mathrm{~mm}$; ; its distance from the perimeter arm is $16 \mathrm{~cm}$. The diameter of the viewing opening is $6 \mathrm{~mm}$. and its distance from the target is 9.5 $\mathrm{cm}$. (3) The perimeter arm may be illuminated with two intensities of light; one carrying the fields well towards the periphery of the retina, the other giving limits narrow enough to fall within the corrected field of the glasses which are worn or may be worn by the patient. This feature provides also for the correction of high astigmatisms, the presence of which make fisld taking annoying and uncertain as a diagnostic procedure. There are other advantages too of providing for the taking of fields at more than one intensity of illumination. (a) Because of the concentric arrangement of the fibers in the nerve trunk and their order of distribution in the retina, it may be of importance as a point of diagnosis to sample the responses of the retina at different degrees of excentricity. And (b) the low illumination fields are in general more sensitive to the influence of the pathologic factors which cause the fields to have different breadths. This is largely due to the fact that low illumination fields are narrow fields. That is, sensitivity falls off gradually near the center of the retina, therefore smaller changes of sensitivity are required near the center of the retina to expand or contract the field. It is probably also due in part to the change produced in the state of the retina's sensitivity at low illumination.

The control of fixation for the presbyopic eye also presents a problem to the perimetrist. The eye with a high degree of preshyopia would have considerable difficulty in seeing with the necessary clearness a fixation object at a distance of $33 \mathrm{~cm}$. By the use of the mirror as fixation control this distance is extended to $66 \mathrm{~cm}$. The satisfactory use of the mirror requires that the image of the eye be seen fairly clearly, and an eye without power of accommodation even if there is no hyperopia for far seeing is approximately 1.50 diopters out of focus for an object at a distance of $6 C \mathrm{~cm}$. When 1.50 diopters out of focus the eye can not see its image in the mirror with a satisfactory degree of clearness. However, the mirror can be used with a fair degree of satisfaction for lesser degrees of presbyopia.

We have three proposals to make for the control of fixation for the presbyopic eye. (1) The use of the mirror for the lesser degrees of presbyopia. (2) The use of an illumination sufficiently low to bring the color fields within the field of the correcting glasses. And (3) the use of a peep-sight or parallax fixation device (Figs. 3 and 4$)^{1}$ similar in principle to the one already described, with the target at a distance great enough to be seen with the necessary degree of clearness by the eye without power of accommodation. Sixty-six cm. has been chosen for this distance because (a) the target can be seen at $66 \mathrm{~cm}$. with sufficient clearness to determine whether or not it is at the center of the viewing opening even tho the eye is as much as 1.50 diopters out of focus; and (b) a greater distance presents difficulty as d matter of feasibility of construction. The device is provicied also with a lens, the distance of which from the target can be varied from its focal length to that which is needed to render the target clearly visible with 3 or more diopters of accommodation. (Three diopters are needed for the perimeter arm). With this lens at its focal length from the target, the rays of light are rendered parallel and the apparent or optical distance of the target is 6 meters. By suitably changing the distance of the lens from the target, the apparent distance of the target can be varied over a wide range. By means of this optical device, therefore, a clear image of the target can be formed on the retina of patients having all degrees of preshyopia with as little disturbance of focus for the perimeter arm for each degree of presbyopia as is possible for that amount of presbyopia. However, as we have already stated, it is quite possible to secure

1. As already stated, the device for control of fixation in high degrees of myopia is also shown in Fig. 3 in position for use. When using either, the other should of course be removed. 
a good control of fixation without the use of the optical attachment.

The fixation device consists of a tube $17.5 \mathrm{~cm}$. long and $8 \mathrm{~mm}$. in diameter, and a light metal rod fastened at one end of the tube and supporting vertically at the other a small plate of hard sheet aluminum $3 \times 3 \mathrm{~cm}$., at the center of which is a disc $4 \mathrm{~mm}$. in diameter. This disc and plate serves as the fixation target. To use this device as a control of fixation the telescope is removed from the tubular axle of the perimeter and the tube of the fixation device is inserted in the axle to a depth of $4.5 \mathrm{~cm}$. To control the depth of this insertion and thus the distance of the target from the eye, the tube is provided with a stop or shoulder $4.5 \mathrm{~cm}$. from the end inserted. The tubular axle and its extension thus form the opening thru which the fixation target is viewed, and when the eye is in position at the center of the perimeter system the target is seen to be centrally located in this opening. The target itself is painted black and the plate at the center of which it is mounted is painted in the same shade of gray as the remainder of the perimeter. The target receives its illumination from the lamp which illuminates the perimeter arm. An unobstructed passage of light from the lamp to the target is possible because of the distance of the target $33 \mathrm{~cm}$. behind the perimeter arm. The lens which is used to vary the optical distance of the target from the eye is mounted in the end of the main tube, facing the target. This tube telescopes to the desired depth into a shorter tube, at the end of which on a short supporting rod is the target.

The steadiness of fixation is greatly influenced by the method of giving the stimulation. One of the serious objections to a moving stimulus is the difficulty of holding a steady fixation while the object to be observed is moving. The alternative procedure is the use of a stationary stimulus. That is, the stimulus is placed at the desired point on the perimeter arm and covered with the preexposure card. The observer takes his fixation and at a given signal the stimulus is exposed and recovered. By this method of giving the stimulation more time is consumed, but a much greater precision of result is possible. A compromise pro- cedure is recommended. That is, the approximate location of the limit is determined with the moving stimulus, and the exact location with the stationary stimulus. By this compromise but very little more time is required, and there is no sacrifice of precision.

In order to provide for the mapping of the normal blind spot and for the quick detection and mapping of central and paracentral scotomata, it has been deemed advisable to add to the perimeter a tangent screen subtending a visual angle of 60 or more degrees. Provision is made so that this screen can be quickly and conveniently attached to the stimulus carriage and moved into position. The stimulus carriage and the tangent screen have at their exact center a circular opening equal in size to the cross section of the tubular aperture about which the perimeter arm rotates. Thus when the tangent screen is in position, $i . e$, with its central point in the axis of rotation of the perimeter arm, the tubular opening in the perimeter is continued thru to the front surface of the tangent screen. This both provides for the exact adjustment of the tangent screen and permits of the convenient use with it of all of the fixation controls which we have described. Cards of white or black as may be desired, with the fields laid off on the tangent scale, are provided for the mapping of the area deficient in the light sense, and of grays of the brightness of the colors for mapping the color deficiencies. This is shown in Fig. 5 . It is quite possible that the stimulus and preexposure cards used for the fieid taking and the larger backgrounds or cards for the tangent screen could be made of thin hard sheet aluminum coated with a flat enamel paint of white, black or grays of the brightness of the colors. If this were done, they could be kept clean by washing and the small paper disc of pigment color used as the stimulus for the field taking is all that would have to be renewed as the result of long periods of use. These paints can be bought in a flat black and a flat white. By using the two in the desired proportions the shades of gray desired may be obtained.

In our own work we have found it convenient to use the large screen just described for a quick survey of the fie!d 
for scotomata, and a smaller screen, similar to the one used to carry the colored stimulus in the field taking, for the actual detailed mapping of the scotomata and the normal blind spot. This screen was made considerably larger than the screens which serve as backgrounds for the colored stimulus in field taking in order that it might serve for the mapping of large scotomata and pathologically enlarged blind spots. It is mounted in a carriage of its own and is shaped to take the curvature of the perimeter arm. This screen is intended not as a substitute for the larger screen, but as a supplement in cases in which such a supplement is found to be convenient and desirable. This device has the following advantages over the large central tangent screen for the actual mapping of the blind areas. (1) It can be moved to any part of the field from the center out to $90^{\circ}$ in any meridian and its center located at the center of the area to be mapped. When the screen is properly centered the mapping can be done as it is on any tangent screen. (2) Upon each screen is drawn 16 meridians running radially from the center of the screen. These meridians are finely graduated so that the limits of sensitivity in any meridian can be read off for the permanent record and transferred to properly planned maps at the convenience of the experimenter. (3) Blind areas are most easily and precisely mapped when the stimulus is made to follow lines radiating from the center of the area. Unless there are such guiding lines, it is difficult to pass from within out or from without in consistently when determining the limits of the blind area, or when checking up the location of a limit by a second or third determination. On a large fixed screen these lines would have to be drawn specially for each scotoma. (4) All of the evidence points toward the importance of mapping the blind areas with colored stimuli, particularly the Mariotte spot. It is highly important that the determinations for the different colors be made on backgrounds of the same brightness as the colors, as will be shown by blind spot studies to be published later. It is much more feasible to arrange for this in case of the smaller movabie fields than in case of a large fixed field. When the background for the smaller field is laid off in graduated radial lines, it can serve for the mapping of many blind spots and scotoma before it need be replaced. In case of a large fixed screen, this superior adaptability and long service would not be possible. (5) With the movable smaller field, it is more nearly possible to map all blind areas under the conditions of equal illumination which obtains in the perimetry of the color fields, than it is in the use of the large fixed screen. In the latter case the illumination at which the mapping is done in one part of the field. may be quite appreciably different from that at which it is done in another part, of the field. The control of illumination while not so important for mapping the blind areas to the light sense stimuli, is very important in the mapping of the blind areas to colors.

With the controls provided in the perimeter recommended, a careful worker can without difficulty reproduce the limits of sensitivity within 1 or 2 degrees.

\section{CONCERNING CONGENITAL COLOBOMA OF THE LENS.}

\section{Walter Bafr Weilder, M.D.}

NEW YORK CITY.

Our present knowledge regarding the condition is cited and a case seen by the author is reported in detail.

Coloboma of the lens is extremely rare, but coloboma of the lens associated with coloboma of the iris or the choroid is a much more frequent occurrence. There is no doubt that this form of congenital defect often escapes detection, because it may exist without any great reduction of vision being present. The case of coloboma of the lens reported in this paper probably never would have been detected except for the atropin used for the refraction.

Kampffer in 1899 was able to collect thirty-two cases of coloboma of the lens, 